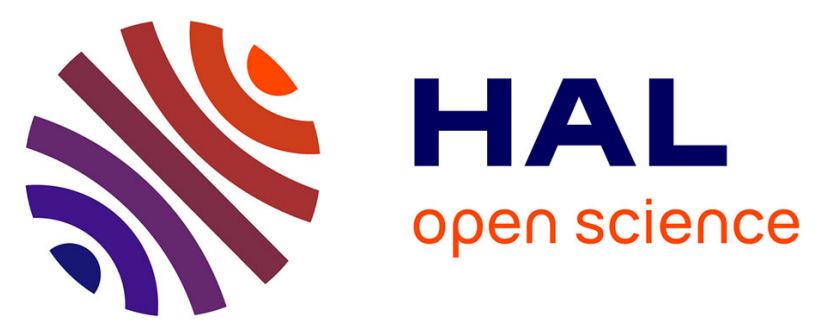

\title{
On an infinite number of quadratures to evaluate beam shape coefficients in generalized Lorenz-Mie theory and Extended Boundary Condition Method for structured EM beams
}

Gérard Gouesbet, L.A. Ambrosio, J.A. Lock

\section{To cite this version:}

Gérard Gouesbet, L.A. Ambrosio, J.A. Lock. On an infinite number of quadratures to evaluate beam shape coefficients in generalized Lorenz-Mie theory and Extended Boundary Condition Method for structured EM beams. Journal of Quantitative Spectroscopy and Radiative Transfer, 2020. hal02453246

\author{
HAL Id: hal-02453246 \\ https://hal.science/hal-02453246
}

Submitted on 21 Dec 2021

HAL is a multi-disciplinary open access archive for the deposit and dissemination of scientific research documents, whether they are published or not. The documents may come from teaching and research institutions in France or abroad, or from public or private research centers.
L'archive ouverte pluridisciplinaire HAL, est destinée au dépôt et à la diffusion de documents scientifiques de niveau recherche, publiés ou non, émanant des établissements d'enseignement et de recherche français ou étrangers, des laboratoires publics ou privés.

\section{(ㄷ)(1) $\$$}

Distributed under a Creative Commons Attribution - NonCommerciall 4.0 International 
Version of Record: https://www.sciencedirect.com/science/article/pii/S0022407319307575

Manuscript_ee64d762104d2d9423c49d605e903af2

\title{
On an infinite number of quadratures to evaluate beam shape coefficients in generalized Lorenz-Mie theory and the Extended Boundary Condition Method for structured EM beams.
}

\author{
Gérard Gouesbet ${ }^{1}$, Leonardo André Ambrosio ${ }^{2}$, James A. Lock ${ }^{3}$ \\ 1.CORIA-UMR 6614- Normandie Université \\ CNRS-Université et INSA de Rouen \\ Campus Universitaire du Madrillet \\ 76800, Saint-Etienne-du Rouvray, France. \\ 2.Department of Electrical and Computer Engineering \\ São Carlos School of Engineering, University of São Paulo

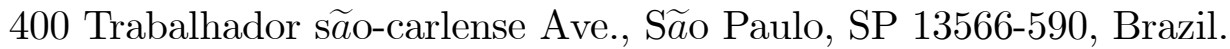 \\ 3.Department of Physics, Cleveland State University, Cleveland, Ohio, 44115, USA. \\ Corresponding author :Gouesbet@coria.fr
}

November 28, 2019

\begin{abstract}
When dealing with light scattering theories such as the T-matrix methods for structured laser beams, e.g. Generalized Lorenz-Mie Theory (GLMT) or the Extended Boundary Condition Method (EBCM), EM fields are expanded over a set of Vector Spherical Wave Functions (VSWFs) involving spherical Bessel functions, with expansion coefficients expressed in terms of Beam Shape Coefficients (BSCs). Although spherical Bessel functions are orthogonal over the range $(-\infty,+\infty)$, the GLMT may be expressed using a non-orthogonal set of spherical Bessel functions defined over $(0,+\infty)$, allowing one to generate an infinite number of quadratures for evaluating the BSCs. This paper points out the difference between orthogonal and non-orthogonal spherical Bessel functions, establishes the infinite number of quadratures and discusses its properties.
\end{abstract}

Keywords : Generalized Lorenz-Mie Theory; Extended Boundary Condition Method; T-matrix; Beam Shape Coefficients; Vector Spherical Wave Functions. 


\section{Introduction.}

The description of electromagnetic structured beams may be carried out in terms of expansions over Vector Spherical Wave Functions (VSWFs), e.g. in the framework of light scattering theories such as Generalized Lorenz-Mie Theory (GLMT) [1], [2], [3] or the Extended Boundary Condition Method (EBCM) [4], [5] for structured beams [6]. In these frameworks, expansion coefficients over the VSWFs can be expressed in terms of sub-coefficients known as Beam Shape Coefficients (BSCs) usually denoted as $g_{n, T M}^{m}$ and $g_{n, T E}^{m}$ (TM: Transverse Magnetic; TE: Transverse Electric) although, originally, BSCs were introduced in the framework of the Bromwich formalism [7]. VSWFs involve spherical Bessel functions which are orthogonal over $(-\infty,+\infty)$. However, the GLMT may be expressed in terms of a non-orthogonal set of spherical Bessel functions defined over the range $(0, \infty)$ instead of being defined over the range $(-\infty,+\infty)$. We examine the implications of the use of a non-orthogonal set of spherical Bessel functions, in particular the fact that BSCs may be evaluated in an infinite number of ways under the form of an infinite number of quadratures, and discuss whether these different ways are equivalent or not. We shall restrict our discussion to the TM-BSCs $g_{n, T M}^{m}$ insofar as the case of TE-BSCs would be treated similarly.

\section{Non-orthogonality of spherical Bessel functions over $(0,+\infty)$ and their consequences.}

In the Bromwich formulation, the TM-BSCs $g_{n, T M}^{m}$ allow one to express the radial component $E_{r}$ of the electric field according to, e.g. Eq.(3.10) in [3]:

$$
E_{r}=E_{0} \sum_{n=1}^{\infty} \sum_{m=-n}^{+n} c_{n}^{p w} g_{n, T M}^{m} \frac{n(n+1)}{r} \Psi_{n}^{(1)}(k r) P_{n}^{|m|}(\cos \theta) \exp (i m \varphi)
$$

in which $P_{n}^{|m|}$ are associated Legendre functions defined according to Hobson's notation, which is also used by Arfken and Weber [8] with an extraprefactor $(-1)^{m}$ introduced, $\Psi_{n}^{(1)}(k r)$ are spherical Bessel functions of the first kind also denoted as $j_{n}(k r)$, and $c_{n}^{p w}$, with $p w$ standing for "plane wave" are plane wave coefficients which occur in the Bromwich formulation of the usual Lorenz-Mie theory [9] and which do not need to be specified in the present paper.

The usual way to isolate the BSCs is as follows. First, use the orthogonality relation for exponentials to get rid of the azimuthal angle $\varphi$ : 


$$
\int_{0}^{2 \pi} \exp \left[i\left(m-m^{\prime}\right) \varphi\right] d \varphi=2 \pi \delta_{m m^{\prime}}
$$

Afterward, use the orthogonality relation for the associated Legendre functions $P_{n}^{m}(\cos \theta)$ to get rid of the polar angle $\theta$ :

$$
\int_{0}^{\pi} P_{n}^{m}(\cos \theta) P_{l}^{m}(\cos \theta) \sin \theta d \theta=\frac{2}{2 n+1} \frac{(n+m) !}{(n-m) !} \delta_{n l}
$$

To take advantage of Eqs.2 and 3, Eq.1 is successively multiplied by integral operators $\int_{0}^{2 \pi}(.) \exp \left(-i m^{\prime} \varphi\right) d \varphi$ and $\int_{0}^{\pi}(.) P_{n^{\prime}}^{|m|} \sin \theta d \theta$ to obtain:

$$
\begin{aligned}
g_{n, T M}^{m}= & \frac{1}{E_{0} c_{n}^{p w}} \frac{2 n+1}{4 \pi n(n+1)} \frac{(n-|m|) !}{(n+|m|) !} \frac{r}{\Psi_{n}^{(1)}(k r)} \\
& \int_{0}^{\pi} \int_{0}^{2 \pi} E_{r} P_{n}^{|m|}(\cos \theta) \exp (-i m \varphi) \sin \theta d \theta d \theta d \varphi
\end{aligned}
$$

It is to be noted that Eq.4 contains an $r$-dependent term $r / \Psi_{n}^{(1)}(k r)$. This does not prevent the BSCs from being complex numbers, as they should, because the integrals in Eq.4 are proportional to an inverse factor $\Psi_{n}^{(1)}(k r) / r$, e.g. [10] for the case of a plane wave propagating along the $z$-axis, [11], [12] for oblique propagation and [13] for any beam perfectly satisfying Maxwell's equations. Furthermore, the double quadrature expression of Eq.4 pertains to what has been call the F1-formulation in [14]. It must also be noted that this F1-formulation does not need any orthogonality property of spherical Bessel functions, and works independently of whether the spherical Bessel functions are orthogonal or not. This is because the only integrals which have to be used in the F1-formulation are the ones over $\theta$ and $\varphi$.

Another way to obtain expressions of BSCs, known as the F2-formulation [14] is by using triple quadratures instead of using double quadratures. For this, we shall use [15], p. 412:

$$
\int_{0}^{\infty} \Psi_{n}^{(1)}(k r) \Psi_{n^{\prime}}^{(1)}(k r) d(k r)=\frac{\pi}{2(2 n+1)} \text { for } n=n^{\prime}
$$


in which the integration interval is from 0 to $\infty$, which is natural since this is the range of the radial variable $r$. Then, rearranging Eq.4 in an obvious way and applying the operator $\int_{0}^{\infty}(.) \Psi_{n^{\prime}}^{(1)}(k r) d(k r)$, we obtain:

$$
\begin{aligned}
g_{n, T M}^{m}= & \frac{(2 n+1)^{2}}{2 \pi^{2} n(n+1) c_{n}^{p w}} \frac{(n-|m|) !}{(n+|m| !} \\
& \int_{0}^{\pi} \int_{0}^{2 \pi} \int_{0}^{\infty} \frac{E r}{E_{0}} r \Psi_{n}^{(1)}(k r) P_{n}^{|m|}(\cos \theta) \exp (-i m \varphi) \sin \theta d \theta d \varphi d(k r)
\end{aligned}
$$

Eqs. 4 and 6 are strictly equivalent when the field descriptions exactly satisfy Maxwell's equations, and provide two different ways to evaluate the BSCs. The first way (double quadratures) does not explicitly eliminate the spherical Bessel functions and therefore does not need to specify whether they constitute a set of orthogonal functions or not. Conversely, the second set explicitly eliminates the spherical Bessel functions. These functions are orthogonal over the range $(-\infty,+\infty)$, according to $[8]$, p. 732 :

$$
\int_{-\infty}^{\infty} \Psi_{n}^{(1)}(k r) \Psi_{n^{\prime}}^{(1)}(k r) d(k r)=\frac{\pi}{2 n+1} \delta_{n n^{\prime}}
$$

However, Eq.5 is defined over $(0,+\infty)$ instead of over $(-\infty,+\infty)$ and is therefore restricted to a non-orthogonal set of spherical Bessel functions. Eq.5 is then to be completed by [15], p. 412:

$$
\int_{0}^{\infty} \Psi_{n}^{(1)}(k r) \Psi_{n^{\prime}}^{(1)}(k r) d(k r)=\frac{\sin \left[\left(n-n^{\prime}\right) \pi / 2\right.}{n(n+1)-n^{\prime}\left(n^{\prime}+1\right)} \text { for } n \neq n^{\prime}
$$

The fact that the spherical Bessel functions $\Psi_{n}^{(1)}(x)$ are not orthogonal over the interval $(0,+\infty)$ is reminiscent of contravariant and covariant components in tensor calculus. For instance, let us consider a point $\mathrm{P}$ in an Euclidean plane spanned by two linear non-orthogonal axes $\mathbf{x}$ and $\mathbf{y}$ crossing with an angle $\gamma \neq 0$ at point $\mathrm{O}$. We may then introduce a vector $\mathbf{V}=\overrightarrow{O P}$ using an intrinsic notation. The vector $\mathbf{V}$ may then receive two different index representations, named the contravariant and the covariant representations. In the contravariant representation, $\mathbf{V}$ is projected onto the axes parallel to them, then defining a contravariant vector $V^{i}=\left(x_{\|}, y_{\|}\right)^{+}$in which $x_{\|}$and $y_{\|}$are the contravariant components of the contravariant vector $V^{i}$, and the cross $(+)$ denotes a 
transpose. Conversely, in the covariant representation, $\mathbf{V}$ is projected onto the axes perpendicularly to them, then defining a covariant vector $V_{i}=\left(x_{\perp}, y_{\perp}\right)$ in which $x_{\perp}$ and $y_{\perp}$ are the covariant components of the covariant vector $V_{i}$. But, because the axes $\mathbf{x}$ and $\mathbf{y}$ are not orthogonal, we have $\left(x_{\|}, y_{\|}\right) \neq\left(x_{\perp}, y_{\perp}\right)$.

Similarly, in the F2-formulation, the BSCs may be viewed as components over a set of basis functions involving non-orthogonal spherical Bessel functions. The analogy with contravariant and covariant vectors, although very loose, was a motivation that led us to a careful examination of uniqueness properties of the BSCs in the framework of the F2-formulation.

Then, let us multiply both sides of Eq.1 by $r$ and apply:

$$
\int_{0}^{\infty} \cdot \Psi_{n^{\prime}}^{(1)}(k r) d k r
$$

to obtain, after using Eq.8, for $n \neq n^{\prime}$ :

$$
\int_{0}^{\infty} r E_{r} \Psi_{n^{\prime}}^{(1)}(k r) d k r=E_{0} \sum_{n=1}^{\infty} \sum_{m=-n}^{+n} c_{n}^{p w} g_{n, T M}^{m} \frac{n(n+1) \sin \left[\left(n-n^{\prime}\right) \pi / 2\right]}{n(n+1)-n^{\prime}\left(n^{\prime}+1\right)} P_{n}^{|m|}(\cos \theta) e^{i m \varphi}
$$

Next, we use Eq.2, to obtain, after a change of $m^{\prime}$ to $m$ :

$$
\frac{1}{2 \pi} \int_{0}^{\infty} \int_{0}^{2 \pi} r E_{r} \Psi_{n^{\prime}}^{(1)}(k r) e^{-i m \varphi} d k r d \varphi=E_{0} \sum_{n=1}^{\infty} c_{n}^{p w} g_{n, T M}^{m} \frac{n(n+1) \sin \left[\left(n-n^{\prime}\right) \pi / 2\right]}{n(n+1)-n^{\prime}\left(n^{\prime}+1\right)} P_{n}^{|m|}(\cos \theta)
$$

Next, we use Eq.3 and, after a change $l \mapsto n$, and rearranging, we obtain for $n \neq n^{\prime}$ :

$$
\begin{aligned}
g_{n, T M}^{m} \sin \left[\left(n-n^{\prime}\right) \pi / 2\right]= & \frac{1}{4 \pi E_{0} c_{n}^{p w}} \frac{n(n+1)-n^{\prime}\left(n^{\prime}+1\right)}{n(n+1)}(2 n+1) \frac{(n-|m|) !}{(n+|m|) !} \\
& \int_{0}^{\infty} \int_{0}^{\pi} \int_{0}^{2 \pi} r E_{r} \Psi_{n^{\prime}}^{(1)}(k r) P_{n}^{|m|}(\cos \theta) \sin \theta e^{-i m \varphi} d k r d \theta d \varphi
\end{aligned}
$$

If $\left(n-n^{\prime}\right)$ is even, i.e. $\left(n-n^{\prime}\right)=2 k$, then $\sin \left[\left(n-n^{\prime}\right) \pi / 2\right]=0$, and Eq.12 implies: 


$$
\int_{0}^{\infty} \int_{0}^{\pi} \int_{0}^{2 \pi} r E_{r} \Psi_{n^{\prime}}^{(1)}(k r) P_{n}^{|m|}(\cos \theta) \sin \theta e^{-i m \varphi} d k r d \theta d \varphi=0 \text { for }\left(n-n^{\prime}\right) \text { even, } n \neq n^{\prime}
$$

Inserting the expression of Eq.1 into the 1.h.s. of Eq.13, and using Eq.8 for $\left(n-n^{\prime}\right)$ even, $n \neq n^{\prime}$, allows one to check the validity of Eq.13 whenever the function $E_{r}(r, \theta, \varphi)$ represents the radial component of an EM field. But, if $\left(n-n^{\prime}\right)$ is odd, i.e. $\left(n-n^{\prime}\right)=2 k+1$, then $\sin \left[\left(n-n^{\prime}\right) \pi / 2\right]= \pm 1$, and Eq.12 leads to:

$$
\begin{aligned}
{\left[g_{n, T M}^{m}\right]_{n \neq n^{\prime}}=} & \frac{1}{4 \pi E_{0} c_{n}^{p w}} \frac{n(n+1)-n^{\prime}\left(n^{\prime}+1\right)}{n(n+1) \sin \left[\left(n-n^{\prime}\right) \pi / 2\right]}(2 n+1) \frac{(n-|m|) !}{(n+|m|) !} \\
& \int_{0}^{\infty} \int_{0}^{\pi} \int_{0}^{2 \pi} r E_{r} \Psi_{n^{\prime}}^{(1)}(k r) P_{n}^{|m|}(\cos \theta) \sin \theta e^{-i m \varphi} d k r d \theta d \varphi \text { for }\left(n-n^{\prime}\right) \text { odd }
\end{aligned}
$$

for a given value of $n$ and with $n^{\prime} \neq n$ being arbitrary, for $\left(n-n^{\prime}\right)$ odd. Thus we appear to have an infinite number of ways to compute BSCs, depending on the value chosen for $n^{\prime}$, as expected from the above-mentioned analogy. With the idea that the F1- and F2-formulations are actually equivalent, and that the set of BSCs is unique, we now have to compare the different sets of BSCs that we obtain, either from Eq.4 or from Eq.14, $\left(n-n^{\prime}\right)$ odd. To do this, we rewrite Eq.14 as:

$$
\begin{aligned}
{\left[g_{n, T M}^{m}\right]_{n \neq n^{\prime}}=} & \frac{1}{4 \pi E_{0} c_{n}^{p w}} \frac{n(n+1)-n^{\prime}\left(n^{\prime}+1\right)}{n(n+1) \sin \left[\left(n-n^{\prime}\right) \pi / 2\right]}(2 n+1) \frac{(n-|m|) !}{(n+|m|) !} \\
& \int_{0}^{\infty} r \Psi_{n^{\prime}}^{(1)}(k r) d(k r)\left\{\int_{0}^{\pi} \int_{0}^{2 \pi} E_{r} P_{n}^{|m|}(\cos \theta) \sin \theta e^{-i m \varphi} d \theta d \varphi\right\} \text { for }\left(n-n^{\prime}\right) \text { odd }
\end{aligned}
$$

The \{.\}-term may however be taken from Eq.4 to obtain:

$$
\left[g_{n, T M}^{m}\right]_{n \neq n^{\prime}}=\frac{n(n+1)-n^{\prime}\left(n^{\prime}+1\right)}{\sin \left[\left(n-n^{\prime}\right) \pi / 2\right]} g_{n, T M}^{m} \int_{0}^{\infty} \Psi_{n^{\prime}}^{(1)}(k r) \Psi_{n}^{(1)}(k r) d(k r)
$$

which by virtue of Eq.8 implies:

$$
\left[g_{n, T M}^{m}\right]_{n \neq n^{\prime}}=g_{n, T M}^{m}
$$


independently of the value of $n^{\prime}\left(n-n^{\prime}\right.$ odd).

TE-BSCs would be treated similarly allowing one to similarly obtain:

$$
\left[g_{n, T E}^{m}\right]_{n \neq n^{\prime}}=g_{n, T E}^{m}
$$

therefore assuring the uniqueness of BSCs.

Strictly speaking, the uniqueness of BSCs has here been demonstrated in the framework of quadrature formulations. The result obtained could have been however expected insofar as the uniqueness of BSCs may be shown to be valid whatever the method used. The demonstration goes on as follows. Let us assume that Eq.1 is valid as well for an alternative set of BSCs $\overline{g_{n, T M}^{m}}$ according to:

$$
E_{r}=E_{0} \sum_{n=1}^{\infty} \sum_{m=-n}^{+n} c_{n}^{p w} \bar{g}_{n, T M}^{m} \frac{n(n+1)}{r} \Psi_{n}^{(1)}(k r) P_{n}^{|m|}(\cos \theta) \exp (i m \varphi)
$$

Hence, by subtraction:

$$
0=E_{0} \sum_{n=1}^{\infty} \sum_{m=-n}^{+n} c_{n}^{p w}\left[g_{n, T M}^{m}-\overline{g_{n, T M}^{m}}\right] \frac{n(n+1)}{r} \Psi_{n}^{(1)}(k r) P_{n}^{|m|}(\cos \theta) \exp (i m \varphi)
$$

Next, applying Eqs.2 and 3 to Eq.20 to successively get rid of the exponentials and of the associated Legendre functions, we obtain:

$$
0=\left[g_{n, T M}^{m}-\overline{g_{n, T M}^{m}}\right] \Psi_{n}^{(1)}(k r)
$$

which exhibits the particular role played by the spherical Bessel functions and implies:

$$
g_{n, T M}^{m}=\overline{g_{n, T M}^{m}}
$$

with a similar demonstration for the TE-coefficicients.

As a final remark, let us note that the present work complements our understanding of the description of structured beams in terms of BSCs, but have no implication concerning the numerical efficiency of the theory. Indeed, in any 
case, triple quadratures of the form of Eq.14 will be more time-consuming than the double quadratures of the form of Eq.4. Let us also take the opportunity of this remark to recall that even the double quadratures of Eq.4 are numerically too time-consuming to constitute an efficient way of evaluating the BSCs, except when they can be analytically solved to obtain closed-form solutions, e.g. for zeroth-order Bessel beams [16], higher-order Bessel beams [17], and superpositions of Bessel beams, either for "frozen waves" [18], [19], [20], [21], or for Mathieu beams which are expressed as well as superpositions of Bessel beams $[22]$.

Otherwise, the arsenal of methods usable to evaluate BSCs also contains localized approximations (with several variants) which may speed up the computations by several orders of magnitude, e.g. [23] to be complemented by [24], [25], [26], and finite series which, after having been forgotten for several decades, has been recently used again due to the limitations encountered when dealing with localized approximations in the case of beams exhibiting axicon angles and/or topological charges, e.g. [27], [28], [29], and references therein. It is also possible to use an angular spectrum decomposition into elementary plane waves either (i) by computing the scattering response of each plane wave and summing up all the responses over the plane waves present in the decomposition, a process which requires the use of GLMT for each tilted plane wave, e.g. [30] or (ii) evaluating the BSCs of each plane wave and summing up to obtain the BSCs of the whole beam, before entering GLMT computations,e.g. without pretending to exhaustiveness [31], [32], [33], [34], [35], [36], [37], [38], [39] and [40] for a variant relying on the use of a Whittaker integral formalism. See also [41] for the relationship between BSCs and plane wave spectra.

\section{Conclusion.}

BSCs in GLMT may be derived by using a non-orthogonal subset of spherical Bessel functions. Motivated by an analogy concerning the difference between contravariant and covariant vectors, we noted that there appears to exist an infinite number of different quadratures to evaluate the BSCs. We have demonstrated that the BSCs obtained by the different ways of evaluating them are identical, so that the decomposition of $E_{r}$ over VSWFs is indeed unique, as expected, and demonstrated in a general framework. As a by-product, we have established a new equation, namely Eq.13.

More generally, the present paper complements our understanding of the structure of the GLMT in the sense that the fact that we may use a nonorthogonal version of spherical Bessel functions by restricting the range of definition of the spherical Bessel functions to $(0,+\infty)$ has not been explicitly noted

previously, nor has it been perceived that it implies the existence of an infinite number of quadratures to evaluate the BSCs.

\section{Funding.}


L.A. Ambrosio thanks FAPESP (S $\widetilde{a}$ o Paulo Research Foundation, project number 2017/10445-0) and CNPq (National Council for Scientific and Technological Development, projects number 426990/2018-8 and 307898/2018-0) for supporting this work.

\section{References}

[1] G. Gouesbet, B. Maheu, and G. Gréhan. Light scattering from a sphere arbitrarily located in a Gaussian beam, using a Bromwich formulation. Journal of the Optical Society of America A, 5,9:1427-1443, 1988.

[2] G. Gouesbet, G. Gréhan, and B. Maheu. Combustion measurements, edited by N. Chigier, chapter : Generalized Lorenz-Mie theory and applications to optical sizing, pages 339-384. Hemisphere Publishing Corporation, NewYork, USA, 1991.

[3] G. Gouesbet and G. Gréhan. Generalized Lorenz-Mie theories, second edition. Springer International Publishing AG, 2017.

[4] P.C. Waterman. Symmetry, unitarity, and geometry in electromagnetic scattering. Physical Review D, 3, 4:825-839, 1971.

[5] M.I. Mishchenko, L.D. Travis, and A.A. Lacis. Scattering, absorption, and emission of light by small particles. Cambridge University Press, Cambridge, 2002.

[6] G. Gouesbet. T-matrix methods for electromagnetic structured beams: A commented reference database for the period 2014-2018. Journal of Quantitative Spectroscopy and Radiative Transfer, 230:247-281, 2019.

[7] G. Gouesbet. T-matrix formulation and generalized Lorenz-Mie theories in spherical coordinates. Optics Communications, 283, 4:517-521, 2010.

[8] G.B. Arfken and H.J. Weber. Mathematical methods for physicists, sixth edition. Elsevier Academic Press, Amsterdam, 2005.

[9] G. Gouesbet and G. Gréhan. Sur la généralisation de la théorie de LorenzMie. Journal of Optics, 13,2:97-103, 1982.

[10] J.A. Lock. Contribution of high-order rainbows to the scattering of a Gaussian laser beam by a spherical particle. Journal of the Optical Society of America A, 10,4:693-706, 1993.

[11] A.A.R. Neves, A. Fontes, L.A. Padilha, E. Rodriguez, C.H. de Brito Cruz, L.C. Barbosa, and C.L. Cesar. Exact partial wave expansion of optical beams with respect to an arbitrary origin. Optics Letters, 31, 16:2477$2479,2006$. 
[12] A.A.R. Neves, L.A. Padilha, A. Fontes, E. Rodriguez, C.H.B. Cruz, L.C. Barbosa, and C.L. Cesar. Analytical results for a Bessel function times Legendre polynomials class integrals. J. Phys. A : Math. Gen., 39:L293L296, 2006.

[13] W.L Moreira, A.A.A. Neves, M.K. Garbos, T.G. Euser, and C.L. Cesar. Expansion of arbitrary electromagnetic fields in terms of vector spherical wave functions. Optics Express, 24, 3:2370-2382, 2016.

[14] G. Gouesbet, C. Letellier, K.F. Ren, and G. Gréhan. Discussion of two quadrature methods of evaluating beam shape coefficients in generalized Lorenz-Mie theory. Applied Optics, 35,9:1537-1542, 1996.

[15] Arfken. Mathematical methods for physicists. Academic Press, London, 1976.

[16] J.A. Lock. Angular spectrum and localized model of Davis-type beam. Journal of the Optical Society of America A, 30, 3:489-500, 2013.

[17] J.J. Wang, T. Wriedt, L.Mädler, Y.P. Han, and P. Hartmann. Multipole expansion of circularly Bessel beams of arbitrary order for scattering calculations. Optics Communications, 387:102-109, 2017.

[18] L.A. Ambrosio. Circularly symmetric frozen waves: Vector approach for light scattering calculations. Journal of Quantitative Spectroscopy and Radiative Transfer, 204:112-119, 2018.

[19] L.A. Ambrosio, M. Zamboni-Rached, and G. Gouesbet. Discrete vector frozen waves in generalized Lorenz-Mie theory: linear, azimuthal and radial polarization. Applied Optics, 57, 12:3293-3300, 2018.

[20] L.A. Ambrosio, M. Zamboni Rached, and G. Gouesbet. Zeroth-order continuous vector frozen waves for light scattering: exact multipole expansion in the generalized Lorenz-Mie theory. Journal of the Optical Society of America B, 36, 1:81-89, 2019.

[21] Leonardo André Ambrosio. Millimeter-structured nondiffracting surface beams. Journal of the Optical Society of America B, 36, 3:638-645, 2019.

[22] A. Chafiq, L.A. Ambrosio, G. Gouesbet, and A. Belafhal. On the validity of the integral localized approximation for on-axis zeroth-order Mathieu beams. Journal of Quantitative Spectroscopy and Radiative Transfer, 204:27-34, 2018.

[23] G. Gouesbet, J.A. Lock, and G. Gréhan. Generalized Lorenz-Mie theories and description of electromagnetic arbitrary shaped beams: localized approximations and localized beam models, a review. Journal of Quantitative Spectroscopy and Radiative Transfer, 112:1-27, 2011. 
[24] G. Gouesbet and J. Lock. Comments on localized and integral localized approximations in spherical coordinates. Journal of Quantitative Spectroscopy and Radiative Transfer, 179:132-136, 2016.

[25] J.J. Wang and G. Gouesbet. Note on the use of localized beam models for light scattering theories in spherical coordinates. Applied Optics, 51, $17: 3832-3836,2012$.

[26] G. Gouesbet. Second modified localized approximation for use in generalized Lorenz-Mie theories and other theories revisited. Journal of the Optical Society of America A, 30, 4:560-564, 2013.

[27] G. Gouesbet, L.F.M. Votto, and L.A. Ambrosio. Finite series expressions to evaluate the beam shape coefficients of a Laguerre-Gauss beam freely propagating. Journal of Quantitative Spectroscopy and Radiative Transfer, 227:12-19, 2019.

[28] L.F.M. Votto, L.A. Ambrosio, and G. Gouesbet. Evaluation of beam shape coefficients of paraxial Laguerre-Gauss beam freely propagating by using three remodeling methods. Journal of Quantitative Spectroscopy and Radiative Transfer, 239:Article 106618 (22 pages), 2019.

[29] G. Gouesbet, L.A. Ambrosio, and L.F.M. Votto. Finite series expressions to evaluate the beam shape coefficients of a Laguerre-Gauss beam focused by a lens in an on-axis configuration. Journal of Quantitative Spectroscopy and Radiative Transfer, 242:Paper 106759, 17 pages, 2019.

[30] G. Gouesbet, J.J. Wang, and Y.P. Han. Transformations of spherical beam shape coefficients in generalized Lorenz-Mie theories through rotations of coordinate system. IV. Plane waves. Optics Communications, 283, 17:32443254, 2010.

[31] A. Doicu and T. Wriedt. Plane wave spectrum of electromagnetic beams. Optics Communications, 136:114-124, 1997.

[32] N.J. Moore and M.A. Alonso. Mie scattering of high numerical aperture fields. In 22nd Congress of the International Commission for Optics : Light for the Development of the World, edited by Ramon Rodriguez-Vera, Rufino Diaz-Uribe, Proceedings of SPIE, Vol 8011, 801162, 2011.

[33] F.G. Mitri, R.X. Li, L.X. Guo, and C.Y. Ding. Resonance scattering of a dielectric sphere illuminated by electromagnetic Bessel non-diffracting (vortex) beams with arbitrary incidence and selective polarization. Annals of Physics, 361:120-147, 2015.

[34] F.G. Mitri, R.X. Li, R.P. Yang, L.X. Guo, and C.Y. Ding. Optical pulling force on a magneto-dielectric Rayleigh sphere in Bessel tractor polarized beams. Journal of Quantitative Spectroscopy and Radiative Transfer, 184:360-381, 2016. 
[35] R. Yang, R. Li, S. Qin, C. Ding, and F.G. Mitri. Direction reversal of the optical spin torque on a Rayleigh absorptive sphere in vector Bessel polarized beams. Journal of Optics, 19:Paper 025602, 17pp, 2016.

[36] F.G. Mitri, R.X. Li, L.X. Guo, and C.Y. Ding. Optical tractor Bessel polarized beams. Journal of Quantitative Spectroscopy and Radiative Transfer, 187:97-115, 2017.

[37] D.P. Chrissoulidis and E. Richalot. Wave-amplitude synthesis applied to Gaussian beam scattering by an off-axis sphere. Journal of the Optical Society of America A, 34, 4:558-567, 2017.

[38] S. Gong, R. Li, Y. Liu, and J. Zhang. Scattering of a vector Bessel vortex beam by a charged sphere. Journal of Quantitative Spectroscopy and Radiative Transfer, 215:13-24, 2018.

[39] Y. Wen, Q. Xi, R. Li, S. Qin, and C. Ding. Scattering of a vector BesselGaussian beam by a sphere. Journal of Quantitative Spectroscopy and Radiative Transfer, 204:165-178, 2018.

[40] A. Chafiq, G. Gouesbet, and A. Belafhal. On the beam shape coefficients of fundamental nondiffracting beam. Journal of Quantitative Spectroscopy and Radiative Transfer, 241:Paper 106750, 9 pages, 2020.

[41] G. Gouesbet and J.A. Lock. On the description of electromagnetic arbitrary shaped beams: The relationship between beam shape coefficients and plane wave spectra. Journal of Quantitative Spectroscopy and Radiative Transfer, 162:18-30, 2015. 